[Contribution trom the Chemical Laboratory of Clart Universtty, No. I, 8.]

\title{
THE CONDUCTANCE OF CONCENTRATED SOLUTIONS OF SODIUM AND POTASSIUM IN LIQUID AMMONIA.
}

By Charles A. Kraus and Walter W. l,ucassa.

Received September 10, 1821.

1. Introduction.

In a series of articles, ${ }^{1}$ it has been shown that the conduction process in solutions of the alkali metals in liquid ammonia is ionic. The positive ion in these solutions is identical with the positive ion of a salt of the same metal, while the negative ion is apparently the negative electron. At low concentrations the negative electron is associated with ammonia, as a consequence of which its speed is of the order of magnitude of that of normal ions; for example, in sodium solutions the speed of the negative ion is 7 times that of the sodium ion. At higher concentrations, however, the carrying capacity of the negative ion increases. This is due to an increase in the speed of this ion in consequence of its loss of ammonia at higher concentrations. At very high concentrations, the speed of the negative ion in ammonia is comparable with that of the ions in metals, since the conductance of the metal solutions in ammonia reaches very high values. In previous investigations the conductance has been measured up to concentrations as high as $2.0 \mathrm{~N}$ with a preliminary observation on the conductance of a saturated solution of sodium in ammonia, the specific conductance of which is approximately one-half that of mercury. It is of interest to examine in detail the conductance curve in the concentrated regions, and, furthermore, to compare the conductance curves of different metals in this region. If the conductance of the concentrated solutions is due to the negative electron, which is therefore the same in solutions of different metals, then we might expect that the conductance curves of different metals in very concentrated solutions will resemble one another quite closely. In the present investigation the conductance of concentrated solutions of sodium and potassium has been studied.

\section{Method, Apparatus and Manipulation.}

Since the conductance of concentrated solutions of the metals in ammonia approaches that of mercury, it is obvious that special means must be adopted in order to measure its value. The ordinary methods, in which a pair of electrodes is introduced into a solution, obviously fail, since the resistance of these solutions is too low to be measured by such means. The apparatus finally employed in this investigation is the same as that employed by Kraus at an earlier date. ${ }^{2}$ This apparatus is shown in outline in Fig.

1 Kralts, This Journal, 30, 1323 (1908); 36, 864 (1914); 43, 749 (1921).

"Krats, ibid., 43, 757 (1921). 
1. The function of its various parts will, perhaps, be best understood by following the procedure in the case of a typical run.

Referring to the figure, Stopcock A connects the apparatus with a high vacuum system. On opening this cock, the entire apparatus, with the exception of the conductivity cell itself, which is shut off by means of the stopcocks B and $\mathrm{C}$, is thoroughly

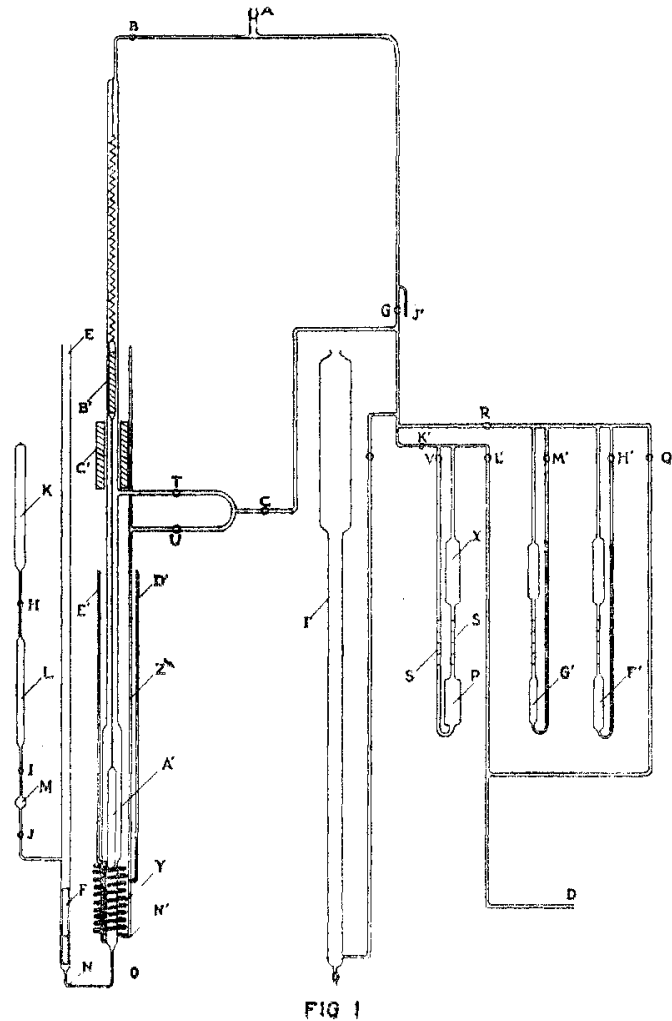

Fig. 1.-Outline of conductivity cell and measuring devices. exhausted. When a sufficiently high vacuum has been obtained, this part of the system is filled with anmonia vapor which passes through $K^{\prime}, L^{\prime}, Q$ and $R$ from a supply cylinder, which is not shown in the figure but which is attached at $D$. The metal, the conductance of whose solutions is to be measured, is freshly cut and rolled into the form of cylinders of suitable size and introduced into the tube $F$ with a glass weight $F$ placed upon it. The tube is then sealed off at $E$ and, after closing the stopcock $G$, the conductance cell is thoroughly evacuated through B. Stopcocks A, B, and $G$ are then closed, $C$ is opened, and the entire system is filled with ammonia from the supply tank. The stopcocks $H, I$ and $\mathrm{J}$ are then closed, trapping ammonia in the tubes $K, I$ and $M$. Stopcock G remaining closed, A and $B$ are opened, thus making connection with the vacuum system. When the conductance cell has been thoroughly evacuated, a bath of paraffin oil is placed around the lower part of the cell and that part of the tube $E$

which contains the metal. The temperature of the bath is raised to about $10^{\circ}$ above the melting point of the metal. When the metal is completely melted, the stopcock $J$ is opened and ammonia is allowed to enter the tube $\mathrm{E}$. Thus there is a slight pressure of ammonia gas in $\mathrm{E}$ which forces the metal through the capillary tube $\mathrm{N}$ into the conductance cell. In most cases this initial pressure is sufficient to force the metal through the capillary, but when this is not so, additional ammonia may be obtained from the chambers $I$ and $K$ and introduced into $F$ in suitable amounts. As a rule, it is well to force the metal over with as small a pressure as possible in order to avoid spattering the molten metal on the upper walls of the cell. When the metal has been transferred, the last traces of ammonia are withdrawn with a pump, and the capillary is sealed off at $O$, while the filling tube $O E$ is removed. When the sealing operation is concluded, the stopcocks $\mathrm{A}$ and $\mathrm{B}$ are shut off and the entire apparatus is filled with ammonia through $\mathrm{C}$.

As was stated above, $D$ is joined to a supply cylinder containing pure ammonia. In 
preparing the solutions in the conductivity cell, ammonia is distilled from the supply cylinder into the measuring cell $P$, which has a capacity of approximately $5 \mathrm{cc}$, A vacuum-jacketed tube containing ammonia is placed around the cell $P$, while the stopcocks $Q$ and $R$ are kept closed and $L^{\prime} V$ and $K^{\prime}$ open. During this operation, connection with the conductance cell s shut off at $\mathrm{C}$ and $\mathrm{G}$. The quantity of ammonia introduced into $P$, and later into the conductance cell, is measured along the scales on the tubes SS. These scales each have a capacity of about $0.5 \mathrm{cc}$. and are graduated in $0.01 \mathrm{cc}$. A third decimal place is obtained by estimating the tenths of the scale divisions. The measuring cells $\mathrm{G}^{\prime}$ and $\mathrm{F}^{\prime}$ are similar, except that one arm of these cells consists of a capillary. $G^{\prime}$ and $F^{\prime}$ have capacities of 1.25 and $2.5 \mathrm{cc}$. respectively.

After determining the volume of liquid in the measuring cell $P$, stopcocks $T, G$ and $V$ are closed and $C$ and $U$ opened, while the ammonia bath surrounding $P$ is removed and replaced by a source of heat. At the same time the conductivity cell is surrounded by a bath of liquid ammonia. Under these conditions, ammonia evaporates in $\mathrm{P}$ and condenses on the metal in the conductivity cell. The purpose of the stopcock $V$ is to force the boiling ammonia through the vaporizing chamber $X$ and also to facilitate the boiling process. Without this stopcock, super-heating is liable to take place and, if the pressure becomes excessive, solvent may be lost either by blowing out one of the stopcocks or by blowing out through the mercury trap $I^{\prime}$.

The initial quantity of approximately $5 \mathrm{cc}$. of ammonia condensed in the conductivity cell is not sufficient to bring the level of the liquid above the opening of the capillary Y. For this purpose, the cell is provided with a displacement cylinder which may be lowered by means of the external solenoid $C$ '. 'The displacer consists of the glass cylinder $\mathrm{A}^{\prime}$ which is connected by a glass rod to a glass-encased soft iron core $\mathrm{B}^{\prime}$ which is suspended by means of a platinum-iridium spring. This device serves to raise the level of the liquid in the conductivity cell as well as to stir the solution in order to insure a unifortm concentration throughout. With the displacement cylinder at its lowest position, the initial charge of ammonia is sufficient to fill the cell to a point where the electrodes are covered. The resistance of the liquid is measured in the glass spiral $Y$, consisting of 14 turns of capillary tubing, one end of which is connected to the cell at the point shown in the figure and the other end of which is connected to the lower extremity of the tube $Z$. One electrode, which consists merely of about $1.5 \mathrm{~mm}$. of platinum wire, is introduced near the lower end of the tube $Z$, while another similar electrode is introduced near the lower end of the main chamber of the conductivity cell. Thus, the current passes through the solution in the cell and through the capillary whose length is approximately one meter and diameter somewhat less than a millimeter. The platinum electrodes are unplatinized, since these solutions do not polarize and platinum black tends to catalyze the reaction between the metal and the solvent. Copper leads are introduced into the wells $\mathrm{D}^{\prime}$ and $\mathrm{E}^{\prime}$ containing mercury, thus making connection with the platinum wires which serve as electrodes.

The resistance was measured by the usual Kohlrausch method, employing a small induction coil or an oscillator as a source of alternating current. The resistance of the lead system was carefully determined beforehand and allowed for in the final calculations.

Due to the difference in diameter of the tube $Z$ and the capillary at $N^{\prime}$, bubbles of ammonia vapor are liable to form at this point. In order to avoid this difficulty at times, it is necessary to close the stopcock $U$ and introduce a slight additional pressure upon the solution through $T$. The ammonia required to furnish this additional pressure is obtained from the measuring cell $\mathrm{P}$, which has been filled in the meantime. The amount of ammonia so introduced is read of the graduated scales SS. When a satisfactory reading of the resistance of the initial solution has been obtained, ammonia is condensed in the cell $G^{\prime}$, the exact amount of liquid condensed being read off on the 
graduated stem. This cell, like cell $\mathrm{P}$, as well as the cell $\mathrm{F}^{\prime}$, is provided with a vaporizing chamber and a control stopcock $\mathrm{M}^{\prime}$, corresponding to $\mathrm{V}$. The method of manipulation is entirely similar to that already described in connection with cell $P$. When a sufficient number of points have been obtained with volume additions of approximately $1.25 \mathrm{cc}$, ammonia is condensed in the cell $\mathrm{F}^{\prime}$, whose volume is approximately $2.5 \mathrm{cc}$. After obtaining a number of observations with $\mathrm{F}^{\prime}$ as measuring cell, the volume of the solution in the conductivity cell becomes sufficiently large so that further additions may be made directly from the cell $P$. The manometer $I$ ' serves to show the pressure under which ammonia is being condensed and also as a manometer for the purpose of determining the vapor pressure of the solutions. When a run is completed, the bath surrounding the conductivity cell is removed and the tip $J^{\prime}$ is broken of, thus allowing the ammonia in the cell to escape. At the same time a small amount of ammonia vapor is passed through $\mathrm{C}$ and $\mathrm{U}$, and through the coil $\mathrm{Y}$. By this means, the capillary is kept free from metal. Without this precaution, the capillary is filled with metal from which it can be freed only with great difficulty. When the ammonia has completely evaporated from the cell through $\mathrm{J}^{\prime}$, this tip is again sealed, stopcocks $\mathrm{C}$ and $\mathrm{G}$ are closed, and the cell is thoroughly evacuated through $B$. When all traces of ammonia vapor have been removed, the tip $\mathrm{J}^{\prime}$ is again broken and the tip $\mathrm{O}$ at the bottom of the cell is likewise broken off. Absolute alcohol, in the case of sodium, and absolute ether, with small additions of absolute alcohol, in the case of potassium, are then drawn into the conductance cell through $O$ by suction applied at $J^{\prime}$. By suitably manipulating the stopcocks $\mathrm{B}, \mathrm{G}, \mathrm{C}$ and $\mathrm{U}$, the solvent may be drawn to any desired height in the cell or the capillary. When the metal has been completely dissolved, the cell is thoroughly washed with distilled water. The original solution of the metal, together with the wash waters, are combined, and the whole is titrated with standard acid.

The measuring cells were calibrated by first weighing empty and then filled with distilled water up to a height noted on the scale. In calculating the amount of ammonia used from cells $\mathrm{F}^{\prime}$ and $\mathrm{G}^{\prime}$, account was taken of the difference in the capillary rise of ammonia and water in the capillaries forming the right arm in each case. The volume of the capillary was also taken into consideration when the relative height of the liquid in the two arms was other than that at which the cell had been calibrated. Correction was also made for ammonia in the vapor space of the conductivity cell when this was found necessary.

The ammonias was run directly into auxiliary cylinders having a capacity of 1 or $2 \mathrm{~kg}$., into which about $3 \mathrm{~g}$. of sodium had previously been introduced. After having withdrawn sufficient ammonia from the auxiliary cylinder so that it was practically free from hydrogen, the small supply cylinder mentioned above was connected to it and from 150 to $200 \mathrm{~g}$. of ammonia was distilled into it. Previously, about $0.25 \mathrm{~g}$. of sodium had been introduced into this cylinder also. As stated, this cylinder was joined to the apparatus at $D$. Thus the solvent was purified by being twice distilled from a sodium solution.

The sodium ${ }^{4}$ was freshly cut before introducing into the tube $\mathrm{E}$. By so doing, and by rapid manipulation, the formation of oxide is avoided. In the case of potassium it was found preferable to cut the metal under light petroleum ether.

One of the most probable sources of inconsistencies in the results is due to the possible lack of uniformity in the concentration of the solutions. Since the resistance changes very rapidly as the concentration changes, it follows that a slight change in the amount of ammonia in the capillary

'Obtained from the National Ammonia Company,

* From the Solvay Process Company. 
$Y$ would cause a considerable change in the measured specific conductance. This source of error was not completely eliminated and, in all likelihood, variations in the measured values obtained are due chiefly to this cause. This error may run as high as $1 \%$ for individual observations.

Since the density of the solutions is not known, the dilution $V$ is expressed in liters of pure solvent per gram atom of metal. As the volume of the ammonia at its boiling point was measured directly, the density enters only in calculating corrections, For this the value 0.674 was assumed.

\section{Calibration of the Conductance Cell.}

The specific conductance of the solutions was determined by direct comparison of the resistance of the solutions with that of mercury. The mercury employed for this purpose was initially cleaned with sand, then with nitric acid, and finally it was twice distilled. The cell, after introducing the mercury, was placed in a bath of water at $20^{\circ}$ and the resistance determined in the usual way. The resistance of the mercury in the capillary, after making correction for the resistance of the leads, was found to be $2.693 \mathrm{ohms}$ at $20^{\circ}$. The specific conductance of the solutions at the boiling point of liquid ammonia was then calculated by means of the equation, $l_{\mathrm{x}}=R^{\prime} l^{\prime} / R_{\mathrm{x}}$, where $l^{\prime}$ is the specific conductance of mercury at $20^{\circ}$, $R^{\prime}$ the resistance of mercury in the cell at $20^{\circ}$, and $R_{\mathrm{x}}$ the resistance of the solution in the cell at $-33.5^{\circ}$. When the cell is placed in liquid ammonia, the leads are at a temperature of $-33.5^{\circ}$ at their lower extremity, while at room temperature at the top. Knowing the resistance of the leads and the temperature coefficient of the metal, correction was made for this effect. The specific conductance of mercury at $20^{\circ}$ was assumed to be $1.0444 \times 10^{4}$.

\section{Experimental Results.}

The results obtained for the conductance of concentrated solutions of sodium and potassium in liquid ammonia are given in Tables $\mathrm{I}$ and II,

\section{TABLE I.}

Specific Conductances of Solutrons of Sodrum in Liouid Ammonia at Various Dilutions AT $-33.5^{\circ}$.

\begin{tabular}{rccccc} 
& Run 2. & & \multicolumn{3}{c}{ Run 3.} \\
No. & $V$. & $l$. & No. & $V$. & $l$. \\
1 & 0.1081 & 5047 & 1 & 0.1626 & 3356 \\
2 & 0.1200 & 5047 & 2 & 0.1698 & 3023 \\
3 & 0.1331 & 4954 & 3 & 0.2099 & 1945 \\
4 & 0.1440 & 4348 & 4 & 0.2510 & 1288 \\
5 & 0.1444 & 4394 & 5 & 0.3210 & 726.2 \\
6 & 0.1560 & 3718 & & & \\
7 & 0.1681 & 3166 & & & \\
8 & 0.1804 & 2687 & & Run 5. & \\
9 & 0.1926 & 2347 & & & \\
10 & 0.2652 & 1174 & No. & V. & $l$. \\
11 & 0.2761 & 1080 & 1 & 0.3463 & 575.3 \\
12 & 0.2768 & 1070 & 2 & 0.4393 & 275.8
\end{tabular}




\begin{tabular}{cccccc}
\multicolumn{7}{c}{ TABL I (continued). } \\
No. & Run 4. & \multicolumn{5}{c}{ Run 6.} \\
1 & $V$. & $l$. & No. & $V$. & $l$. \\
2 & 0.1616 & 3167 & 1 & 0.3393 & 612.8 \\
3 & 0.2042 & 1981 & 2 & 0.4262 & 297.6 \\
4 & 0.2470 & 1344 & 3 & 0.5099 & 148.3 \\
5 & 0.3230 & 714.4 & 4 & 0.5933 & 79.56 \\
6 & 0.4011 & 379.9 & 5 & 0.7612 & 20.21 \\
7 & 0.5772 & 92.19 & 6 & 0.9265 & 5.988 \\
8 & 0.7527 & 22.95 & 7 & 1.298 & 1.269 \\
& 0.9263 & 6.493 & 8 & 1.674 & 0.6456
\end{tabular}

respectively. At the top of each sub-table is given the number of the run. In the first column is given the number of the point in the run; in the second column, the dilution $V$ in liters of solvent per gram atom of metal; and, in the third column, the specific conductance in reciprocal ohms. A number of runs, which were obviously in error, have been omitted.

TABLE II.

Specific Conductances of Solutions of Potassium in Lroutd Ammonia at Various DIIUTIONS AT $-33.5^{\circ}$.

\begin{tabular}{cccccc} 
& Run 1. & & \multicolumn{3}{c}{ Run 2. } \\
No. & $V$. & $l$. & No. & $V$. & $l$. \\
1 & 0.1572 & 2940 & 1 & 0.0853 & 4569 \\
2 & 0.2004 & 1858 & 2 & 0.1058 & 4560 \\
3 & 0.2418 & 1288 & 3 & 0.1295 & 4190 \\
4 & 0.3157 & 701.4 & 4 & 0.1519 & 3233 \\
5 & 0.3891 & 389.8 & 5 & 0.1730 & 2571 \\
6 & 0.5577 & 91.16 & 6 & 0.2103 & 1772 \\
7 & 0.7229 & 21.69 & 7 & 0.2466 & 1278 \\
& & & 8 & 0.3317 & 640.7 \\
& & & 9 & 0.4194 & 318.8
\end{tabular}

5. Discussion.

In Fig. 2 are shown values of the logarithms of the specific conductance of sodium solutions plotted as ordinates against values of the logarithms of the dilution plotted as abscissas. The values of Run 2 are represented by circles, those of Run 3 by crosses, those of Run 4 by combined circles and crosses, those of Run 5 by squares and those of Run 6 by broken crosses. In order to compare the results here obtained with those in the more dilute solutions previously measured by Kraus, a few values from the earlier investigation have been added on the figure, being represented as broken vertical lines. As may be seen, the two are in excellent agreement. In Fig. 3 the heavy curve passing through the points is that of potassium solutions, while that of sodium solutions is represented as a lighter line. Here, again, the results of Run 1 are represented by çircles and those of Run 2 by crosses, while the earlier values of Kraus are represented by broken vertical lines. 
The first few points on the curve at the highest concentrations lie on a horizontal straight line. These points give the conductance of solutions saturated with the metal. The points of intersection between the horizontal portions and the other branches of the curves represent saturated solutions. The concentrations of the saturated solutions may therefore be obtained from these curves. On making the necessary calculations

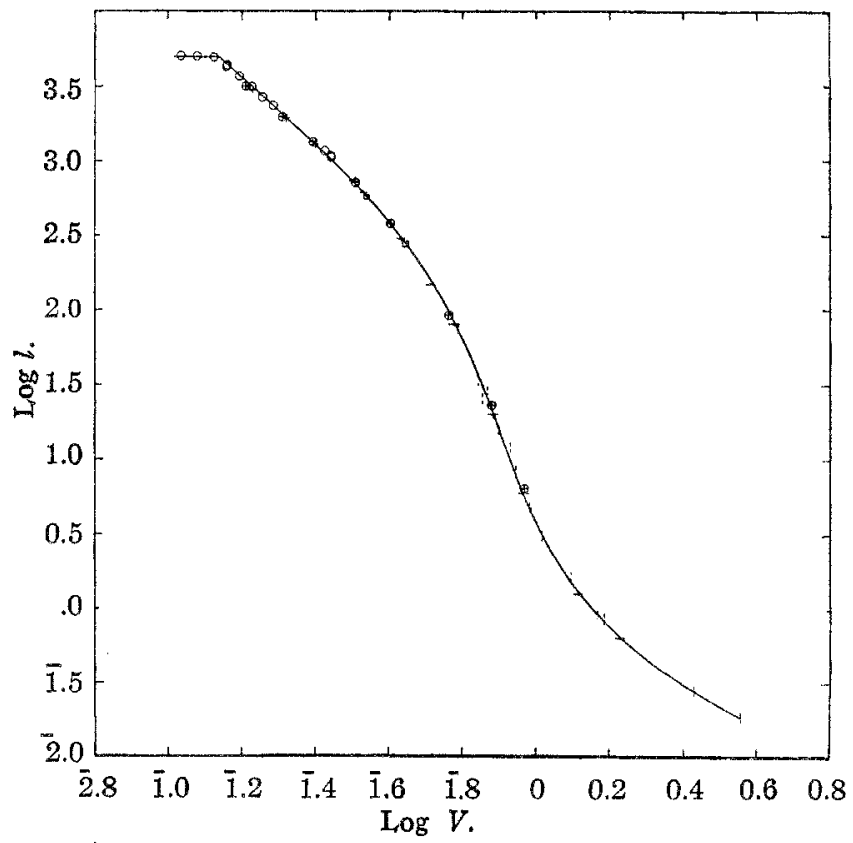

Fig. 2.-Conductance curve for solutions of sodium in ammonia.

it is found that one atom of sodium is dissolved in 5.367 molecules of ammonia, and that one atom of potassium is dissolved in 4.866 molecules of ammonia. The agreement with the determinations of Ruff and Geisel ${ }^{5}$ is fairly good. Taking into account the difficulties involved in the direct determination of the solubility by the method of Ruff and Geisel, it would appear that the present results are the more reliable.

The value obtained for the specific conductance of the more concentrated solutions of sodium and potassium in liquid ammonia is a very striking result. The specific conductance of a saturated sodium solution is 5047.0, while that of a saturated potassium solution is 4569 . The sodium solutions conduct slightly better than the potassium solutions. The specific conductance of mercury at $20^{\circ}$ is $1.044 \times 10^{4}$ from which it is evident that the value of the conductance of these solutions is comparable with that of the metals. However, in making such a comparison the values of the

- Ruff and Geisel, Ber., 39, 828 (1906). 
atomic, rather than those of the specific, conductance should be compared. In Table III are given values of the atomic conductance of various metals at $0^{\circ}$ or near room temperatures. ${ }^{\circ}$

TABLE III.

ATOMTC CONDUCTANCES OF METALS.

\begin{tabular}{|c|c|c|c|}
\hline Metal. & $\begin{array}{l}\text { Atomic } \\
\text { conduct- } \\
\text { ance. } \\
\mathbf{X} \times 10^{-} \text {. }\end{array}$ & Metal. & $\begin{array}{l}\text { Atomic } \\
\text { conduct- } \\
\text { ance. } \\
\Lambda \times 10^{-6} \text {. }\end{array}$ \\
\hline$\ldots \ldots \ldots \ldots \ldots \ldots$ & 6.999 & Tantalum..... & 1.339 \\
\hline Potassium,$\ldots \ldots \ldots \ldots \ldots \ldots$ & 6.503 & $\ldots \ldots \ldots \ldots$ & 1.252 \\
\hline Sodiutu.................. & 5.288 & Osmium..... & 1.119 \\
\hline 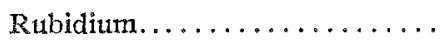 & 4.845 & Tellurium ${ }^{a} \ldots$ & 1.052 \\
\hline Copper.................. & 4.559 & Thallium... & 0.9778 \\
\hline 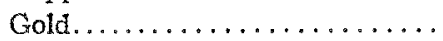 & 4.547 & Nickel................... & 0.9613 \\
\hline Cesium.................. & 3.898 & 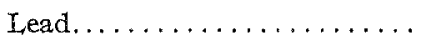 & 0.9222 \\
\hline Aluminum $\ldots \ldots \ldots \ldots \ldots \ldots$ & 3.834 & 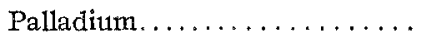 & 0.9082 \\
\hline Titanium $\left(\mathrm{N}_{2}\right) \ldots \ldots \ldots \ldots \ldots$ & 3.345 & Platinum............... & 0.8314 \\
\hline Magnesium............... & 3.215 & Iron. $\ldots \ldots \ldots \ldots \ldots \ldots \ldots$ & 0.8031 \\
\hline Chromium................. & 2.989 & Strontium $^{a} \ldots \ldots \ldots \ldots \ldots \ldots$ & 0.7194 \\
\hline Calcium, $99.5 \% \ldots \ldots \ldots \ldots$ & 2.457 & Cobalt, $99.8 \% \ldots \ldots \ldots$ & 0.7064 \\
\hline 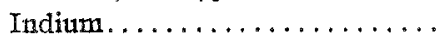 & 1.905 & Manganese $\left(\mathbb{N}_{2}\right) \ldots \ldots \ldots$ & 0.6561 \\
\hline Cadmium.................. & 1.875 & 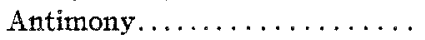 & 0.4658 \\
\hline Rhodium.................. & 1.811 & Arsenic .................. & 0.3735 \\
\hline Zinc................. & 1.713 & Gallium. . & 0.2208 \\
\hline Lithium.................... & 1.534 & Bismuth. . & 0.1972 \\
\hline 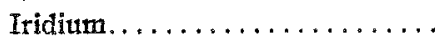 & 1.414 & Mercury........... & 0.1564 \\
\hline & & Saturated sodium sol ${ }^{b} \ldots \ldots \ldots$ & 0.6720 \\
\hline
\end{tabular}

a Calculated on the assumption that the conductance of silver amounts to 60 $\times 10^{4}$.

${ }^{b}$ Calculated on the assumption that the density of the solution is equal to that of the pure solvent. The density is actually less than that of the solvent. The value given for $\Lambda$ is therefore less than the true one.

The atomic conductance of a saturated solution of sodium in liquid ammonia is $0.672 \times 10^{6}$. It is evident that the atomic conductance of saturated solutions of sodium and potassium in liquid ammonia is of the order of that of such metals as strontium and iron, and much greater than that of mercury and bismuth. If the conductance measurements could be extended into the supersaturated regions, it is evident, from the form of the curves, that much larger values of the atomic conductance might be expected. It is interesting to note that, as may be seen from the table, those elements which exhibit the highest atomic conductance all belong to the first group of elements. Among the elements having an atomic conductance greater than $1.5 \times 10^{6}$, all belong to the first three groups of elements, with the exception of rhodium, chromium and titanium.

The manner in which the conductance varies as a function of the con-

" Lanđolt-Börnstein "Physikalisch-Chemische Tabellen," 1912. 
centration is very interesting. At the highest concentrations, the specific conductance increases approximately five fold, when the concentration is doubled. In this region, the relation between the specific conductance and the concentration may be expressed by an exponential equation, since the logarithm of the specific conductance varies very nearly as a linear function of the logarithtn of the dilution, as may be seen from Fig. 2. At somewhat lower concentrations, the conductance curve is concave toward the axis of concentrations, after which it exhibits an inflexion point at about $V=0.65$. In this region the specific conductance of the solution increases enormously with the concentration. Between $V=0.5933$ and

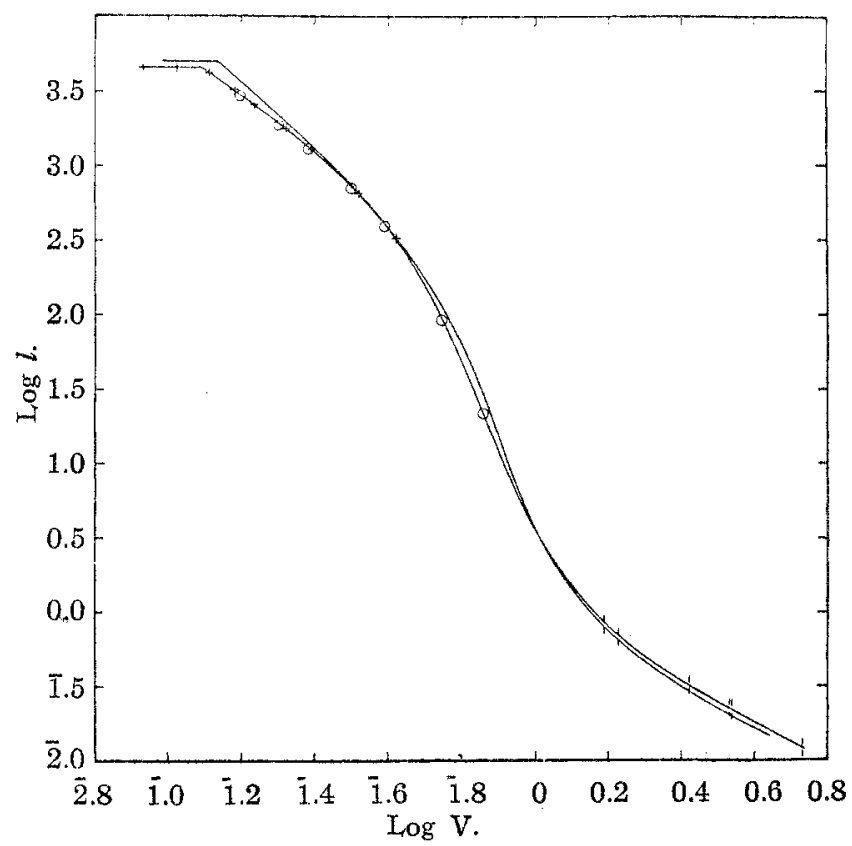

Fig. 3.-Comparison of conductance curves for potassium and sodium in ammonia.

$V=0.3393$ the conductance increases from 79.56 to 612.8 or nearly 10 fold, while between $V=1.298$ and $V=0.5933$ the conductance increases from 1.269 to 79.56 or more than 60 -fold. In the neighborhood of $V=$ 0.67 a concentration increase of $1 \%$ is accompanied by a conductance increase of approximately $15 \%$. It is in these regions that the negative electrons are becoming free from the ammonia molecules with which they are associated in the more dilute solutions. At higher concentrations this factor is doubtless of smaller influence, and it is possible that a change in the total number of carriers present has a material influence on the conductance.

Very striking, is the great similarity of the curves for sodium and potas- 
sium. Indeed, they almost coincide over a considerable range of the concentration interval. In the most concentrated solutions the conductance of potassium is somewhat lower than that of sodium. The two curves then coincide, after which again they diverge slightly at a value $\log V=$ 1.7. Thereafter they again intersect and diverge slightly at lower concentrations owing to the greater conductance of the potassium ${ }^{7}$ ions. Apparently, the specific conductance of the more concentrated sodium and potassium solutions is very nearly the same at the same equivalent concentration. When we take into account the fact that the densities of the two solutions probably vary appreciably ${ }^{8}$ and that, furthermore, the solvation of the positive ions in all likelihood differs for the two metals, it is remarkable that the two curves should approach each other so closely. This is merely an expression of the fundamental fact that the conductance of these solutions is due to the same cause; namely, to the negative electron. It would appear from this, that both the number of electrons and the speed with which they move in these solutions is a function primarily of the equivalent concentration. Since it has previously been shown that the negative carrier in dilute solutions of different metals in ammonia is the same, and since it has been shown that the increase in conductance with increasing concentration is due to the increased speed of the negative carriers in these solutions, it follows that the conductance of the concentrated solutions is due to a common negative carrier, obviously the negative electron. Moreover, since the order of the conductance of these solution is the same as that of the metals, the conclusion appears justified that the conduction process in metals consists essentially in a motion of the negative electron.

\section{Summary.}

1. The specific conductance of solutions of sodium and potassium in liquid ammonia at its boiling point has been measured from concentrations in the neighborhood of normal up to the saturation point.

2. As the concentration increases, the specific conductance increases enormously, reaching the values $0.5047 \times 10^{4}$ and $0.4569 \times 10^{4}$ for saturated solutions of sodium and potassium respectively.

7 Kraus, Triss Journal, 43, 749 (1921).

${ }^{8}$ In respect to density, these solutions are most exceptional. While no exact quantitative data have yet been obtained, the order of magnitude of the volume change may be seen from the following observation. The level of the solution at the third point in the second run with potassium was noted and, when the run was completed, the volume of the cell up to this height was obtained roughly by filling it with water. The amount of metal was of course known and the volume of ammonia at this point was likewise known. From these data the density of this solution was calculated to be 0.632 . How abnormal these solutions are is realized on noting that $2.48 \mathrm{~g}$. of a solid of density 0.86 and $5.55 \mathrm{~g}$. of a liquid of density 0.674 form a solution of density 0.632 . 
3. The specific conductance of solutions of sodium and potassium at the same equivalent concentration is very nearly the same.

4. The equivalent conductance of saturated solutions or sodium and potassium in liquid ammonia is of the order of magnitude of that of such metals as strontium and iron.

5. The solubility of sodium and potassium in liquid ammonia at its boiling point has been found to be 5.367 and 4.866 mols of ammonia per atom for sodium and potassium respectively.

6. The results of this investigation furnish further evidence in support of the hypothesis that the conduction process in metals consists in a motion of negative carriers of sub-atomic dimensions, which carriers are the same for all metals.

WORCESTER, MASSAChUSETTS.

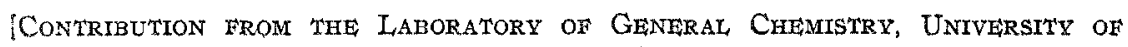
WISCONSTN.]

\section{THE PREPARATION AND PROPERTIES OF THE PERSULFIDES OF HYDROGEN.}

By JaMES H. WALTON AND LI,EWEIIXN B. PARSONS.

Received September 20, 1921.

Introduction.

When an excess of hydrochloric acid is added to a solution of a polysulfide of an alkali or one of the alkaline earths, a yellow oily liquid separates. Since the time of Scheele, who first prepared this substance, much work has been done on its preparation and the study of its properties. Among the more recent investigators, the work of Rebs, ${ }^{1}$ Bruni and Borgo, ${ }^{2}$ and Bloch and Höhn ${ }^{3}$ are worthy of note. The experiments of the latter authors are the most important in connection with this investigation. These investigators prepared the yellow oil by dissolving sulfur in a solution of sodium sulfide contained in a flask through which a stream of hydrogen was passed to prevent oxidation. The resulting solution was chilled and then run into a hydrochloric acid mixture consisting of one part of hydrochloric acid and one part of ice, surrounded by a mixture of ice and salt. Operating in this way they obtained a product with a density varying from 1.62 to 1.69 . The yellow oil when subjected to fractional distillation, yielded two products which on analysis gave the empirical formulas $\mathrm{H}_{2} \mathrm{~S}_{2}$ and $\mathrm{H}_{2} \mathrm{~S}_{8}$. Further experiments confirming the work of Bloch and Höhn were carried out by Schenck and Falcke. ${ }^{4}$

${ }^{1}$ Rebs, Ann. Chem., 246, 356 (1888).

a Bruni and. Borgo, Gazz. chim. ital., 38, 279, 292 (1907-8).

Bloch and Höhn, Ber., 41, 1961 (1908).

4 Schenck and Falcke, Ber., 41, 2600 (1908). 